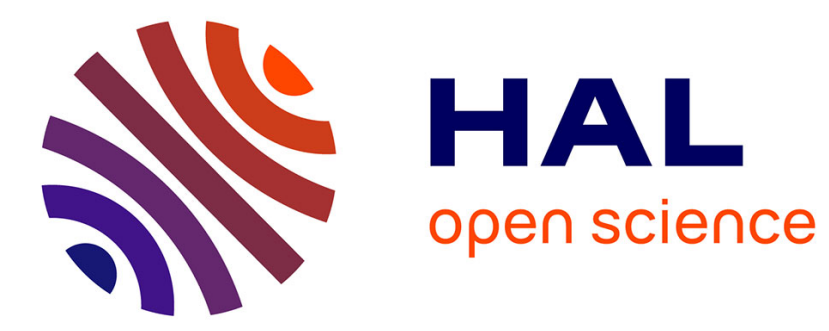

\title{
Notes sur le Bakong et son implantation \\ Christophe Pottier
}

\section{To cite this version:}

Christophe Pottier. Notes sur le Bakong et son implantation. Bulletin de l'Ecole française d'ExtrêmeOrient, 1996, 83 (1), pp.318-326. 10.3406/befeo.1996.3792 . halshs-01989077

\section{HAL Id: halshs-01989077 https://shs.hal.science/halshs-01989077}

Submitted on 22 Jan 2019

HAL is a multi-disciplinary open access archive for the deposit and dissemination of scientific research documents, whether they are published or not. The documents may come from teaching and research institutions in France or abroad, or from public or private research centers.
L'archive ouverte pluridisciplinaire HAL, est destinée au dépôt et à la diffusion de documents scientifiques de niveau recherche, publiés ou non, émanant des établissements d'enseignement et de recherche français ou étrangers, des laboratoires publics ou privés.

\section{()ㅜ) $\Theta$}

Distributed under a Creative Commons Attribution - NoDerivatives| 4.0 International 


\section{Notes sur le Bakong et son implantation}

Christophe Pottier

\section{Citer ce document / Cite this document :}

Pottier Christophe. Notes sur le Bakong et son implantation. In: Bulletin de l'Ecole française d'Extrême-Orient. Tome 83, 1996. pp. 318-326;

doi : https://doi.org/10.3406/befeo.1996.3792

https://www.persee.fr/doc/befeo_0336-1519_1996_num_83_1_3792

Fichier pdf généré le 17/01/2019 


\section{CAMBODGE}

\section{Notes sur le Bakong et son implantation}

Dans le cadre de recherches sur l'occupation du territoire angkorien et de l'élaboration d'une nouvelle carte archéologique de la région d'Angkor, la photodétection nous a permis de repérer de nombreux sites inédits ${ }^{1}$.Par ailleurs, l'analyse des clichés aériens récents ${ }^{2}$ peut parfois fournir des renseignements complémentaires sur les sites connus. C'est le cas du Bakong, à Roluos (cf. figure 1), et les lignes qui suivent présentent certains éléments inédits qui permettent de reconsidérer la chronologie d'implantation de la capitale d'Indravarman connue par les sources épigraphiques ${ }^{3}$.

\section{Un réseau de bassins dans l'enceinte extérieure}

Du Bakong, outre la pyramide et les édifices des deux premières enceintes, on connaît les sanctuaires de briques et de grès qui se répartissent dans la troisième enceinte (enceinte extérieure) ${ }^{4}$. Mais les photographies aériennes (cf. photographie 1$)^{5}$ montrent aussi la présence d'un réseau complet de bassins carrés ${ }^{6}$. Les traces de huit bassins de 60 mètres de côté ponctuent régulièrement les faces intérieures de la troisième enceinte à laquelle ils sont accolés, chaque bassin étant situé approximativement dans le prolongement du second mur d'enceinte (cf. figure 4).

\section{Un réseau de bassins de part et d'autre de la chaussée orientale}

À l'extérieur de la troisième enceinte, la photodétection permet aussi le repérage de deux alignements de bassins à cent mètres de part et d'autre de l'axe oriental. Au sud de la chaussée orientale ${ }^{7}$, à cent mètres de la douve extérieure, la première série présente une succession de

1. Nos travaux de préparation d'une carte archéologique d'Angkor ont fait l'objet d'un mémoire de DEA soutenu en juin 1993 sous la direction de B. Dagens, Université de la Sorbonne Nouvelle, Paris III (cf. Pottier, 1994). L'abréviation « $\mathrm{CP}$ » utilisée pour la numérotation des sites inédits ou non encore inventoriés renvoie à la liste dressée pour ce mémoire.

2. Les travaux de photodétection étaient essentiellement fondés sur une couverture aérienne réalisée par FINMAP en février 1992 et tirée au $25000^{e}$.

3. Les signes diacritiques n'ont pas été employés et nous avons gardé une orthographe usuelle des noms propres khmers, estimant que ce choix ne saurait interférer avec la compréhension du présent article.

4. Lunet de Lajonquière, 1911,p. 265-269 et fig. 77. Ces sanctuaires ont été dégagés, relevés et décrits en 1943 et 1944 par M. Glaize.

5. La reproduction du tirage FINMAP a aimablement été réalisée par M. Ravaux, photographe.

6. On trouve la trace de quatre bassins de forme ovoïde sur la carte topographique IGN/EFEO au $10000^{\mathrm{e}}$ (Dumarçay-Pottier, 1993. pl. 15 et 16). Sur le terrain, les bassins sont, pour la plupart, encore en eau.

7. La chaussée orientale apparaît comme surélevée sur une largeur de $60 \mathrm{~m}$ et sur environ $700 \mathrm{~m}$ de long. 
cinq bassins carrés espacés régulièrement d'est en ouest ${ }^{1}$. Chaque bassin mesure environ $60 \times 60 \mathrm{~m}$, l'intervalle entre chacun a la même dimension. Ces bassins se succèdent donc sur une longueur totale de $540 \mathrm{~m}$. Sur le terrain, ces bassins encore en eau ne révèlent aucune trace de gradins en latérite ou en grès. Le second alignement est situé symétriquement au nord de la chaussée orientale ${ }^{2}$. Seul le bassin occidental (le plus proche de la douve extérieure du Bakong) n'a pas de symétrique parfait au nord. Sur l'emplacement de son vis-à-vis est situé, légèrement décalé vers le nord-ouest, le Srah Andaung Preng CP $364^{3}$, un vestige de plan sensiblement carré différent et plus large $(100 \times 95 \mathrm{~m})$. Sa configuration est assez originale : il est constitué d'une levée de terre qui ceinture un bassin carré de soixante mètres de côté dont le centre est occupé par un tertre légèrement rectangulaire $(25 \times 32 \mathrm{~m})$. Au milieu de celui-ci est situé un dernier bassin rectangulaire où quelques blocs de latérite qui affleurent, suggèrent la présence de gradins parementés ${ }^{4}$. Accolé à la face orientale de ce vestige, un trapeang (bassin creusé rectangulaire) englobe dans sa moitié sud les quatre bassins de la série nord ${ }^{5}$. L'incorporation des bassins dans ce trapeang montre le caractère évidemment postérieur de celui-ci et elle explique pourquoi les formes des quatre bassins nord sont actuellement moins claires que celles de leurs symétriques au sud. Enfin, CP364 et le trapeang étant liés, on peut en déduire que le cinquième bassin, à l'extrême est, a probablement disparu sous CP364.

Ces indications montrent que deux alignements de bassins bordaient la chaussée orientale extérieure du Bakong. Postérieurement, le vestige CP364 et son trapeang ont recouvert les bassins au nord de la chaussée (cf. figure 4).

\section{Un réseau de bassins de part et d'autre de la chaussée septentrionale}

Nous n'avons pas trouvé de traces d'ensembles de bassins aux chaussées sud et ouest du Bakong. Les axes des chaussées y sont à peine visibles. À l'ouest, l'axe semble obliquer légèrement vers le sud et se continuer par une digue-canal qui rejoint le Prasat Beng au sud de Siemreap. H. Marchal suggère qu'il ait même pu se prolonger jusqu'au Prasat Chedei ${ }^{6}$. Au sud, la chaussée n'est guère visible jusqu'à Prei Monti, mais son prolongement rejoint à la hauteur du Prasat Kok Dong le large canal qui descend plein sud pendant quinze kilomètres vers le Tonlé Sap et le village flottant Phoum Kan Trâp (cf. figure 1).

1. Ces bassins sont identifiables sur la carte topographique au $10000^{\circ}$ (Dumarçay-Pottier, 1993. pl. 16).

2. Notons que ce système de bassins peut être mis en parallèle avec ceux qui bordent la chaussée orientale extérieure du Phnom Bakheng, dégagés et relevés au $10000^{\circ}$ en février 1934 par M. Périnelli du Service Géographique. H. Marchal note à ce sujet : On pourra remarquer l'abondance des trapân qui sont portés sur cette carte, encore de l'aveu même de Monsieur Périnelli beaucoup n'ont-ils pas pu être relevés faute de temps car la plupart se dissimulaient sous une brousse impénétrable (...). Ce qui frappe à la première inspection du relevé de M. Périnelli, c'est la régularité de l'alignement d'un grand nombre de trapân à proximité ou en bordure des grandes avenues partant de la base du Bakheng dans l'axe même du temple (Rapport de la Conservation d'Angkor, janvier et février 1934).

3. Srah Andaung Preng: «le bassin de la fosse ancestrale».

4. Le bassin principal $(70 \times 60 \mathrm{~m})$ est en décaissé de $1,6 \mathrm{~m}$, le bassin central environ d'un mètre. Ce dernier mesure $11 \mathrm{~m}$ nord-sud sur $18 \mathrm{~m}$ est-ouest. Ce vestige a conservé une topographie très nette bien qu'il serve depuis peu de potager. Une chaussée relie actuellement le sud de l'îlot central à la digue périphérique; elle nous a semblé être récente.

5. Une levée de terre encore visible ceinture ce trapeang rectangulaire de $490 \mathrm{~m}$ est-ouest sur $120 \mathrm{~m}$ nord-sud. La limite septentrionale des quatre bassins correspond à l'axe oriental de ce trapeang. Cet axe est identique à celui du vestige CP 364 . Le trapeang semble évidemment être lié à ce vestige.

6. «Dans cette région, nous avons remarqué très nettement une voie allant de Bakon vers le lac du Tonlé Sap et de direction nord-sud. Une autre voie part de Bakon en direction ouest pour aboutir au Vat Chay Dey » (Rapport de la Conservation d'Angkor, juillet 1936). 
Mais on observe trois bassins carrés similaires (même distance de la chaussée, même taille) encore nettement visibles le long de la chaussée septentrionale, au nord de la douve extérieure (cf. figure 4). Ces trois bassins feraient-ils partie d'un complexe similaire à celui de l'est, réalisé sur cette chaussée nord? L'analyse des photographies aériennes et les reconnaissances de terrain semblent bien l'indiquer.

Sur le côté oriental de la chaussée nord, les deux bassins méridionaux sont encore très nets et en eau durant la majeure partie de l'année. Celui du milieu est en partie perceptible malgré le tertre informe qui le chevauche en partie. Son voisin au nord est invisible. On note qu'il se situerait sous la chaussée orientale de Prah Kô. De même pour le bassin extrême-nord dont l'emplacement est occupé par un vestige Srah Andaung Preng CP 520, étonnamment semblable au CP 364 du même nom mentionné plus haut ${ }^{1}$. La coïncidence est remarquable puisque, outre le nom, ces deux vestiges possèdent le même type de plan très singulier et des dimensions similaires ${ }^{2}$. On note aussi qu'ils sont tous les deux situés au nord-est d'un temple, à l'amorce de leur chaussée orientale (celle du Bakong pour CP364, celle de Prah Kô pour CP520). Ces deux vestiges proviennent donc vraisemblablement d'un même projet.

À l'ouest de la chaussée axiale, le bassin extrême-sud est parfaitement visible et encore en eau. Son voisin au nord est encore aisément repérable dans le décrochement particulier qu'il provoque dans la douve sud de Prah Kô. Les trois bassins septentrionaux ne sont pas visibles. Ils seraient situés dans l'enceinte extérieure de ce temple. Les multiples similitudes avec le système de bassins de la chaussée orientale, les traces collectées à la chaussée nord, leur dimension, leur rythme et leur symétrie confirment bien l'existence d'un système identique mais plus altéré, cinq bassins manquants actuellement. On note que la configuration actuelle du second bassin sud du côté ouest ne saurait être compréhensible que par son antériorité par rapport à Prah $\mathrm{Ko}^{3}$. Celle-ci justifie l'altération importante du système de bassins et, en particulier, la disparition des trois bassins plus au nord du côté ouest sous des terrassements liés à la construction de l'enceinte de Prah Kô.

\section{Problème de chronologie relative entre Bakong et Prah Kô}

Nous observons donc trois réseaux de bassins: dans la troisième enceinte et le long des chaussées extérieures est et nord. L'état des deux derniers montre qu'ils ont subi une altération partielle par Prah Kô, CP 364 et CP 520. Ceci suggère l'antériorité des chaussées sur Prah Kô, et donc un aménagement centré sur l'emplacement du Bakong préexistant à l'implantation de Prah Kô.

Les documents épigraphiques contredisent en apparence ce schéma. Ils présentent une chronologie précise des fondations réalisées par Indravarman: Indratataka (877 de notre ère), Prah Kô (879) puis Bakong (881) (Cœdès, 1989, p. 206-207). C'est d'ailleurs grâce à la précision de ces dates que $\mathrm{Ph}$. Stern a pu dégager un premier modèle de diversité et rythme des fondations royales khmères. Prah Kô est considéré comme antérieur à Bakong.

Nous reviendrons donc brièvement sur les indications fournies par les inscriptions. Les dates pour Prah Kô et Bakong correspondent à la consécration de ces temples, alors que celle

1. H. Marchal, reprenant la rédaction des rapports de la Conservation d'Angkor après le décès de G. Trouvé, mentionnait en juillet 1935 «à une centaine de mètres au Nord-Est du Gopura III Est (de Prah Kô), un petit srah carré dont les bords sont maçonnés en latérite : ce srah est connu par les indigènes sous le nom de Srah Andaun Pren ». Le localisant par erreur «à l'intérieur du fossé extérieur qui entoure Pra Kô», Marchal n'a pas relevé la présence d'une douve autour du bassin.

2. Les dimensions du Srah Andaung Preng CP 520 sont légèrement plus importantes : $120 \times 110 \mathrm{~m}$ extérieur et $90 \times 80 \mathrm{~m}$ pour le bassin. Au milieu de l'îlot central $(50 \times 45 \mathrm{~m})$, le bassin $(20 \times 15 \mathrm{~m})$ présente aussi des traces de gradins en latérite. Aucune trace de chaussée n'est visible. existante.

3. On imaginerait difficilement pour quelles raisons un bassin serait creusé à cheval sur une douve 
de l'Indratataka indique le début des travaux. Cette date provient d'une interprétation de la stance VII de la stèle de Prah Kô (K. 713) qui mentionne: Des qu'il (Indravarman) eut reçu le pouvoir royal, il fit cette promesse: "Dans cinq jours, à partir d'aujourd'hui, je commencerai à creuser, etc.» (Cœdès, 1937: 25). Il s'agissait donc bien d'un bassin conclut Ph. Stern en l'associant à l'Indratataka (Stern, 1954, p. 663).

On rappellera cependant que cette stance offre aussi une autre interprétation. G. Groslier y voit une allusion à la date de construction de Prah Kô : que pourrait signifier en effet, je commencerai à creuser, etc., sinon à "bâtir un temple», ce que Barth et Bergaigne ont compris et ont écrit dans leur traduction (G. Groslier, 1935, p. 177) ${ }^{1}$. La construction d'un temple khmer commençant avec le creusement d'une fosse de fondation, cette seconde interprétation semble d'autant plus plausible que le même vers se retrouve dans les inscriptions des linteaux des tours centrales (K. 310) et nord-est (K. 317) de Prah Kô qui font directement référence aux divinités installées dans ce temple, et sur les piédroits sud de la tour sud côté est (K. 304) et tour ouest côté nord (K. 308) à Bakong. Il semble alors que cette stance peut faire allusion à la construction des temples de Prah Kô et Bakong ${ }^{2}$. P. Stern note d'ailleurs que ces temples ont été sans doute commencés simultanément (Stern, 1954, p. 664). On peut donc envisager le début des travaux de Prah Kô, du Bakong et du baray en 877 de notre ère. L'Indratataka n'aurait été que l'ouvrage le plus rapidement fini. Il semble dès lors qu'Indravarman ait mis en œuvre très rapidement, et simultanément, les divers éléments d'un plan global d'aménagement de la cité de Hariharalaya. Cependant, à moins d'imaginer une grossière erreur fort improbable de l'urbaniste d'Indravarman, la contemporanéité des deux temples ne saurait toujours pas expliquer l'altération de la chaussée septentrionale du Bakong.

Les sources épigraphiques sont malheureusement muettes sur le détail des réalisations de Jayavarman II et de Jayavarman III. Ces lacunes ont poussé certains à considérer cette période comme pauvre en réalisations majeures, ou même à y voir l'indice d'une certaine faiblesse $(P$. Dupont, 1952, p. 166-168). La rareté des renseignements épigraphiques ne saurait cependant condamner ces deux règnes à l'inactivité. Cl. Jacques, rappelant que «l'argumentum a silentio n'a strictement aucune valeur» considère au contraire que Jayavarman II «s'est montré capable d'accomplir une œuvre civilisatrice importante» (Jacques, 1972, p. 219). Le même auteur précise que l'épigraphie mentionne ce roi occupant de nombreux centres autour d'Angkor et fondant même la ville d'Amarendrapura qui n'a malheureusement pas encore pu être identifiée. Il note que «les travaux d'hydraulique n'étaient pas étrangers à ces soucis» et qu'il «serait très surprenant qu'il n'ait rien entrepris par la suite » ${ }^{3}$. J. Dumarçay a d'ailleurs récemment proposé de n'attribuer à Indravarman que la réalisation de la digue septentrionale du baray de Lolei, les trois autres digues formant un réservoir d'inspiration javanaise (du type «waduk») ayant été créées par Jayavarman II (Dumarçay, 1994: 217-219, fig. 3). Enfin, «les constructions de Jayavarman II sont assez nombreuses et jalonnent presque toutes ses résidences » (Groslier, 1961, p. 88), dont la région de Roluos ${ }^{4}$. La quantité et la variété des sanctuaires réalisés au Kulen montrent au moins le dynamisme qu'a pu connaître la construction à cette époque.

$\mathrm{Cl}$. Jacques souligne que «l'on connaît mal d'où le roi Indravarman tirait ses droits à la couronne de l'empire khmer» et émet l'hypothèse d'une «période de flottement» entre les

1. G. Groslier relève que cette interprétation donne aussi directement la durée de construction du temple de Prah Kô, soit deux ans.

2. Un dernier élément est venu récemment accréditer indirectement notre hypothèse : $\mathrm{Cl}$. Jacques mentionne que la stance VII précise littéralement «Dans cinq jours à partir d'aujourd'hui, je commencerai ce qui commence par un creusement» (Jacques, 1995).

3. La stèle du Prasat Ben (K. 989) mentionne le creusage d'un bassin à Indrapura entrepris durant le règne de Jayavarman II (Jacques, 1972, p. 219).

4. On citera brièvement les Prasat Kuk Dong, Trapeang Pong, Kok Pô, Svay Prahm, Olok, Kandal Dom Sud, Kaek et Kuk Trun. Ces sanctuaires présentent aux moins des traces antérieures au règne d'Indravarman (Stern, 1938, p. 182-189). 
règnes de Jayavarman III et d'Indravarman (Jacques, 1972, p. 217). Il reste qu'Indravarman décide de se rattacher à la «tradition » du culte du Devaraja établie par Jayavarman II (Dupont, 1952 , p. 175). On note aussi qu'il choisit de demeurer comme son prédécesseur à Hariharalaya, cité occupée déjà depuis plus d'un siècle. Il y consacre des sanctuaires pour ses ancêtres et ses prédécesseurs (à Prah Kô pour Jayavarman II/Parameçvara et à Bakong pour Jayavarman III/Vishnuloka). Comme ses prédécesseurs, il effectue diverses reprises et modifications sur des temples antérieurs. Il reconnaît aussi l'authenticité des attributions de terres effectuées par Jayavarman II ${ }^{1}$. Dans cette continuité qu'Indravarman revendique, et si on considère l'hypothèse d'une "période de flottement" d'interrègne, l'engagement (et l'empressement) du nouveau roi à engager des travaux cinq jours après son sacre pourrait alors être considéré comme une assurance de sa volonté de poursuivre des travaux interrompus à la disparition de Jayavarman III.

Quoi qu'il en soit, on notera que les règnes de Jayavarman II et de Jayavarman III ne furent sans doute pas particulièrement pauvres. De même, le règne d'Indravarman revêt un certain caractère de continuité, au moins dans le choix de la capitale. Enfin, l'incertitude est générale sur le détail d'une occupation de la zone de Bakong avant $881^{2}$. La contradiction entre les données épigraphiques et l'existence d'un état antérieur du Bakong n'est donc qu'apparente. D'ailleurs, G. Codès avait suggéré que le Bakong «par sa forme en pyramide, indique un temple du dieu-roi (ayant) pu être commencé par Jayavarman II à son retour du Phnom Kulên pour servir au nouveau culte royal " (Cœedès, 1938, p. 40-48 et 1992, p. 281 282). Des recherches sur les monuments de la première enceinte pourraient peut-être fournir d'autres arguments à cette hypothèse, mais cela dépasse le cadre de notre présente étude. Sans avancer une telle hypothèse, nous proposerons l'existence d'un état antérieur du Bakong uniquement basé sur les traces relevées par photodétection, sans y inclure les édifices du Bakong tel que nous les connaissons actuellement. Cet état comprendrait donc au moins l'implantation de l'enceinte extérieure, les axes et le système de bassins le long des chaussées extérieures. Les similitudes du vocabulaire de cette composition suggèrent que le réseau de bassin de la troisième enceinte puisse être aussi rattaché à ce premier état (cf. figures 2 et 3 ).

Le premier état que nous proposons présente une configuration logique et équilibrée qui renforce la centralité (douve et bassins) de la composition pendant que les systèmes de bassins

1. «Il semble qu'Indravarman Ier, en reconnaissant comme valable la restauration de la monarchie tentée antérieurement par Jayavarman II, ait validé ipso facto les donations que ce roi avait pu faire » (Dupont, 1952, p. 176).

2. On relèvera que $M$. Glaize conclut son rapport annuel de 1944 en précisant à propos des édifices de la troisième enceinte du Bakong qu'il venait de dégager : «Si les travaux n'ont rien révélé de bien remarquable au point de vue architectural, ils ont confirmé que les divers sanctuaires étaient de même style que la pyramide, contemporains ou légèrement antérieurs en date ». On remarquera cependant que la suggestion d'une éventuelle antériorité repose sur le recoupement de quelques rares détails relevés par Glaize lors des dégagements et des recherches qu'il effectua au cours des travaux de restauration qu'il dirigea au Bakong de 1937 à 1944. En août 1943, Glaize note que la tour $n^{\circ} 9$ présente deux colonnettes qui «paraissent intermédiaires entre celles du Phnom Kûlen et celles de (Prah Kô) ou les autres trouvées en d'autres points de Bakong». Une frise trouvée lors des dégagements de cette même tour lui semble aussi «légèrement plus ancienne» que celles du style de Prah Kô. À la tour $n^{\circ} 7$, Glaize met au jour une statue de Vishnu (DCA 4351) qui lui «paraît d'un style intermédiaire entre celui du Phnom Kûlen et celui de Prah Kô (...). Les similitudes avec la statue $n^{\circ} 3.105$ du Prasat Olok voisin sont évidentes, et les deux pièces semblent être l'œuvre du même artiste " (Rapport de la Conservation d'Angkor, septembre 1943). Il semble cependant que cette statue correspond au style de Prah Kô (Alvares, 1992, p. 31). Enfin, Glaize a remarqué que les gopura est et ouest de deuxième enceinte ont leur base recouverte par le remblai des chaussées franchissant la douve, et que le mur de seconde enceinte est aussi masqué sous le même remblai. Il serait tentant d'y voir la trace d'un état antérieur, où les axes et l'enceinte auraient déjà été déterminés. 
des chaussées extérieures marquent, avec un langage identique, l'importance des axes ${ }^{1}$. La photodétection montre qu'ultérieurement, ce plan fut oblitéré par l'implantation de Prah Kô, de CP 520 et de CP 364 et son trapeang. Rien ne prouve actuellement que Prah Kô et CP 520 sont contemporains. L'altération du plan originel de Bakong a donc pu se faire en plusieurs étapes. Mais le positionnement privilégié de CP 520 au nord-est semble assujetti à l'ordonnancement de Prah Kô. Sa ressemblance avec le CP 364 laisse penser que ce dernier serait alors aussi contemporain et que ces deux vestiges proviennent du seul projet de réaménagement de la capitale Hariharalaya élaboré par Indravarman lors de son accession au trône. Le premier état du Bakong aurait donc été conçu par ses prédécesseurs dans la première moitié du IX ${ }^{\mathrm{e}}$ siècle, Indravarman se contentant d'inscrire dans un plan existant la majorité des édifices que nous trouvons actuellement dans les trois enceintes et d'édifier Prah Kô dans l'un des rares espaces (presque) libres. À la recherche de la capitale de Jayavarman II à Hariharalaya, G. Cœdès proposait deux possibilités pour situer la résidence royale: Prei Monti ou Prah Kô (Cœdès, 1989, p. 282). Si la chronologie que nous proposons est exacte, l'enceinte de Prei Monti pourrait offrir l'emplacement du Palais de Jayavarman II ou de Jayavarman III, l'enceinte extérieure de Prah Kô celui d'Indravarman.

\section{Christophe POTTIER}

\section{BIBLIOGRAPHIE}

\section{ALVARES, $\mathrm{S}$.}

1992 "L'iconographie du temple du Bakong de sa fondation à la fin du XIIIe siècle", Histoire de l'art, 20, Paris, p. 27-38.

CCEDÈS, G.

1937 Inscriptions du Cambodge, vol. I, Publ. EFEO, Hanoi.

1938 «Le fondateur de la royauté angkorienne et les récentes découvertes archéologiques au Phnom Kulên », CEFEO, 14, p. 40-48. Réimpression de l'EFEO, Articles sur le Pays Khmer, vol. II, Paris, 1992, p. 281-282.

1989 Les états hindouisés d'Indochine et d'Indonésie, Paris.

DAGENS, $\mathrm{B}$.

1994 «Le temple indien en Asie du Sud-Est, Archéologie d'une forme», Recherches nouvelles sur le Cambodge, Études thématiques 1, EFEO, Paris, p. 259-272.

DUMARÇAY, J.

1994 «Histoire des retenues d'eau khmères», Journal Asiatique CCLXXII.1, p. 371389.

DUMARÇAY, J. et POTTIER, Ch.

1993 Documents topographiques de la Conservation d'Angkor, Mémoires Archéologiques XXI, EFEO, Paris.

DUPONT, P.

1952/54 «Études sur l'Indochine ancienne, Les débuts de la royauté angkorienne», BEFEO XLVI, fasc. 1, Paris, p. 119-176.

1. Alors que la présence d'une chaussée monumentale orientale est assez évidente, on peut s'interroger sur le rôle d'une chaussée nord alors que l'Indratataka n'est pas encore censé être réalisé. L'existence d'un réservoir de type «waduk », créé par Jayavarman II et transformé ultérieurement en baray par Indravarman, expliquerait alors le caractère monumental de la chaussée septentrionale du premier état du Bakong. 
GLAIZE, $M$.

1936/44 Rapports de la Conservation d'Angkor.

GROSLIER, B. Ph.

1961 Indochine, carrefour des arts, Paris.

1979 «La cité hydraulique angkorienne: exploitation ou surexploitation du sol?», $B E F E O$ LXVI, Paris, p. 161-202.

GROSLIER, G.

1935 «Troisièmes recherches sur les cambodgiens», BEFEO XXXV, Hanoi, p. 160206.

JACQUES, C.

1970 «Études d'épigraphie cambodgienne - IV. Deux inscriptions du Phnom Bakhen (K. 464 et K. 558). - V. La stèle du Prasat Cha Chouk (K. 1034) », BEFEO LVII, Paris, p. 57-90.

1971 «Études d'épigraphie cambodgienne - VI. Sur les données chronologiques de la stèle de Tûol Ta Pec (K. 834)», BEFEO LVIII, Paris, p. 163-176.

1972 «Études d'épigraphie cambodgienne - VII. Sur l'emplacement de royaume d'Aninditapura. - VIII. La carrière de Jayavarman II », BEFEO LIX, Paris, p.193220.

1976 «Études d'épigraphie cambodgienne - IX. La stèle du Baphûon, K. 583 », $B E F E O$ LXIII, Paris, p.351-368.

1978 «Études d'épigraphie cambodgienne - XI. Autour de quelques toponymes de l'inscription de Prasat Trapan Run K. 598: La capitale angkorienne de Yasovarman Ier à Suryavarman Ier ", BEFEO LXV, Paris, p.281-321.

1991 Angkor, Paris.

1995 «L'eau et l'épigraphie», Angkor et l'eau, Actes du Colloque International de Siemreap, 28-30 juin 1995, Phnom Penh, à paraître.

LUNET DE LAJONQUIÈRE, É.

1911 Inventaire descriptif des monuments du Cambodge, vol. III, Paris.

MARCHAL, $\mathrm{H}$.

1934 /36 Rapports de la Conservation d'Angkor.

POTTIER, Ch.

1994 «Élaboration d'une carte archéologique de la région d'Angkor», Actes de la $5 e$ Conférence Internationale de l'European Association of Southeast Asian Archaeologists, Paris, à paraître.

STERN, Ph.

1938 «Le style des Kulên», BEFEO XXXVIII, Paris, p.111-149.

1938 «Travaux exécutés au Phnom Kulên», BEFEO XXXVIII, Paris, p.151-171.

1938 «Hariharalaya et Indrapura », BEFEO XXXVIII, Paris, p.175-197.

1954 «Diversité et rythme des fondations royales khmères », BEFEO XLVII, Paris, p.649-687. 


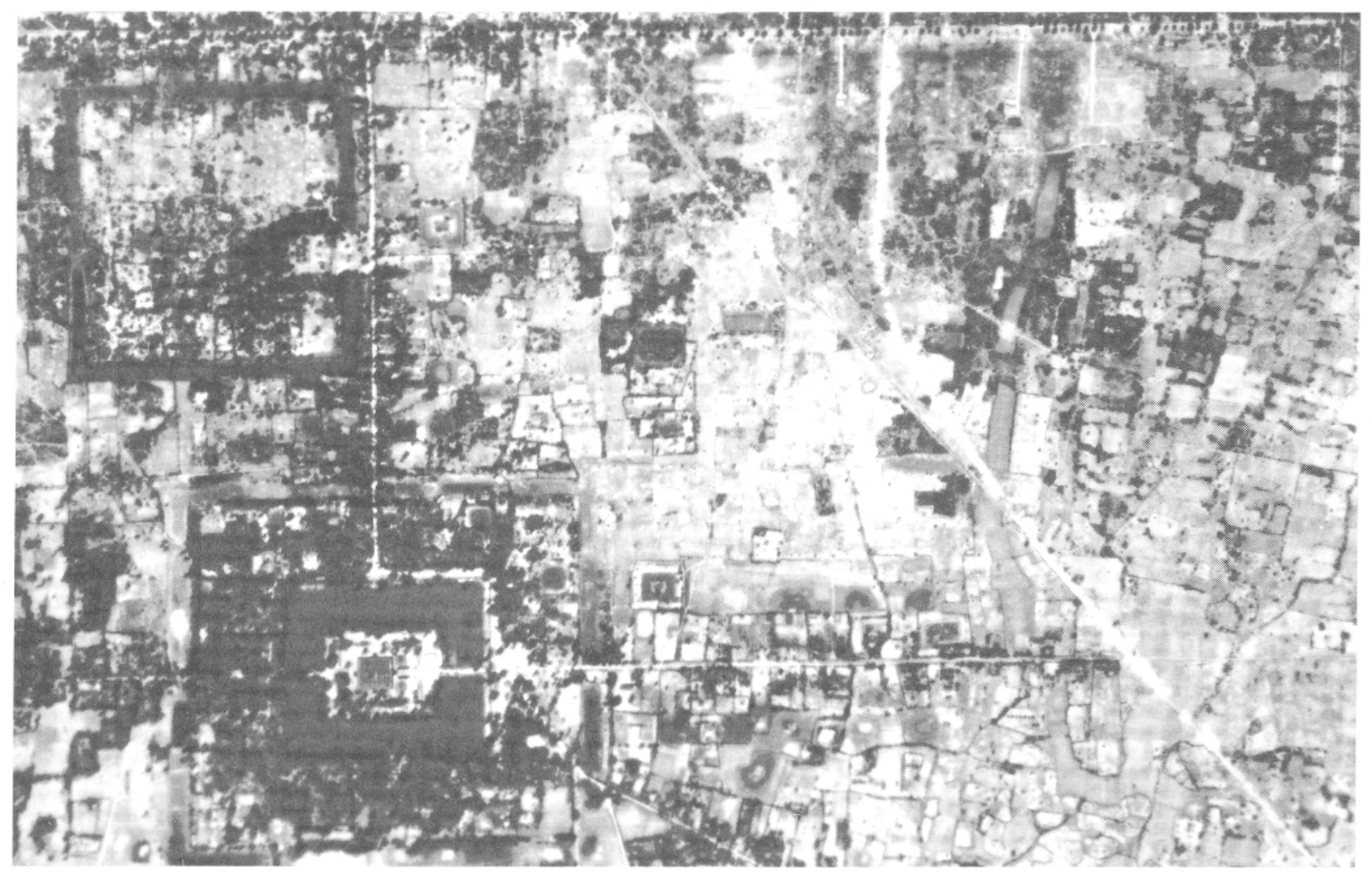

Bakong, photographie aérienne FINMAP, février 1992 ( $\mathrm{n}^{\circ} 1078$, bande 35).

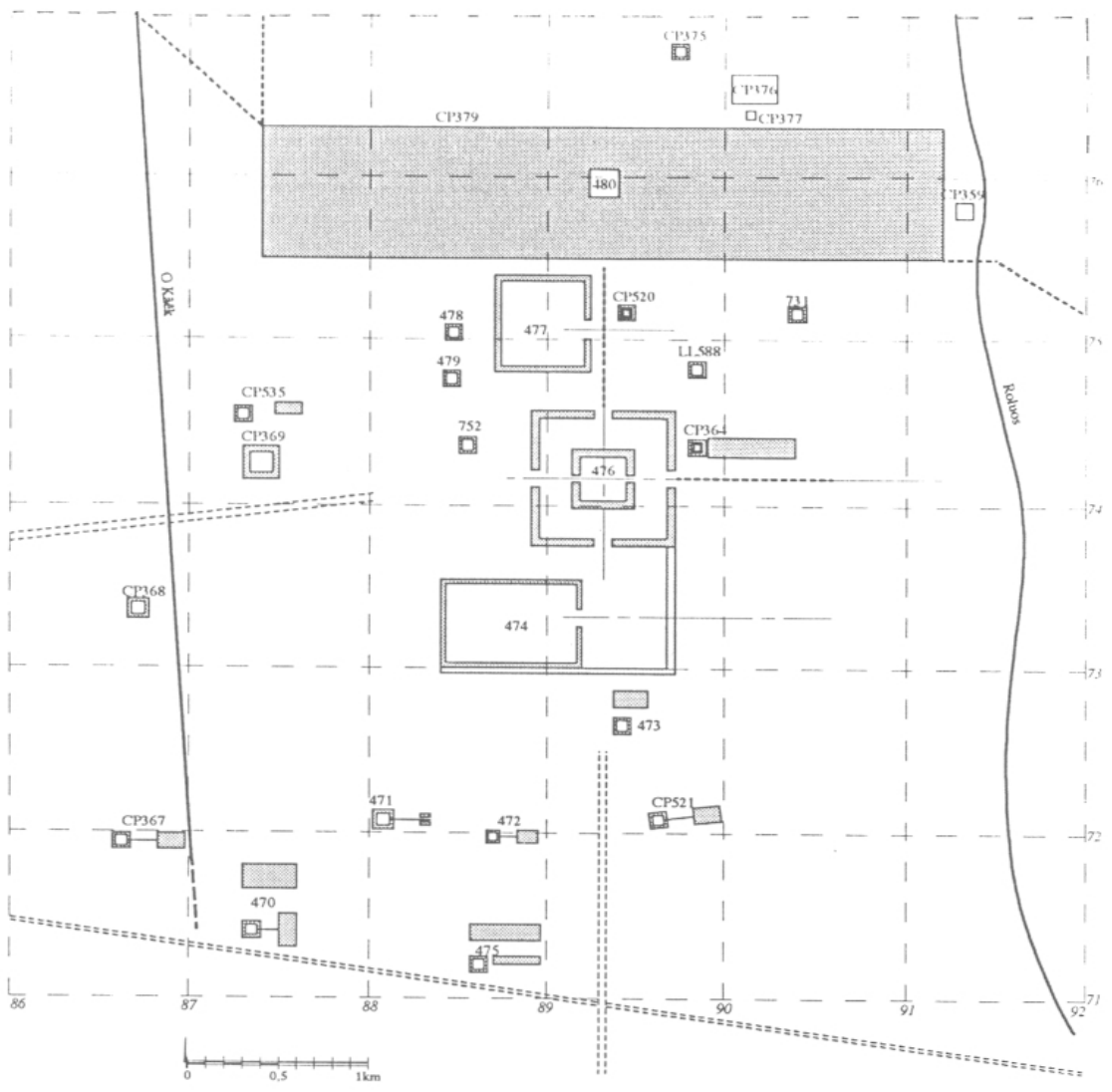

fig.1 : Sites repérés dans la zone de Roluos. 


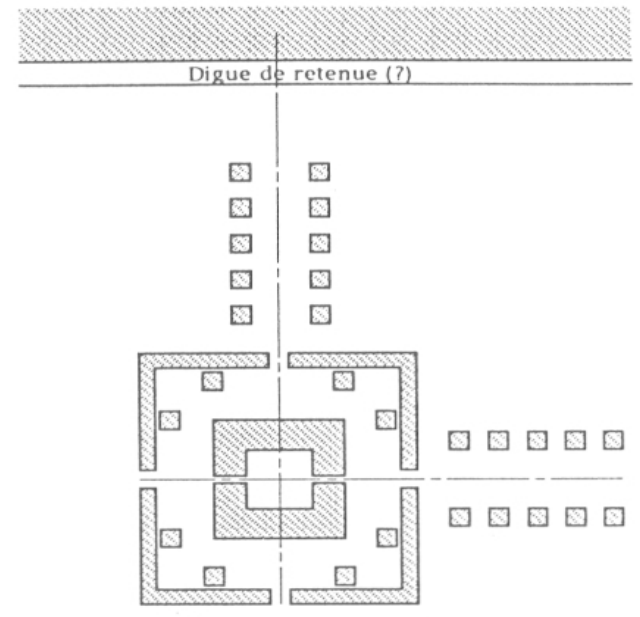

fig. 2 : Bakong, premier état.

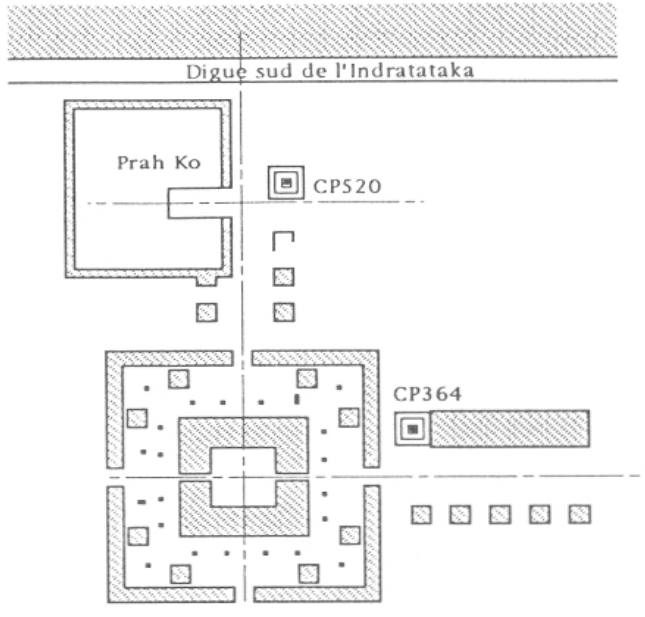

fig. 3: Bakong, état sous Indravarman.

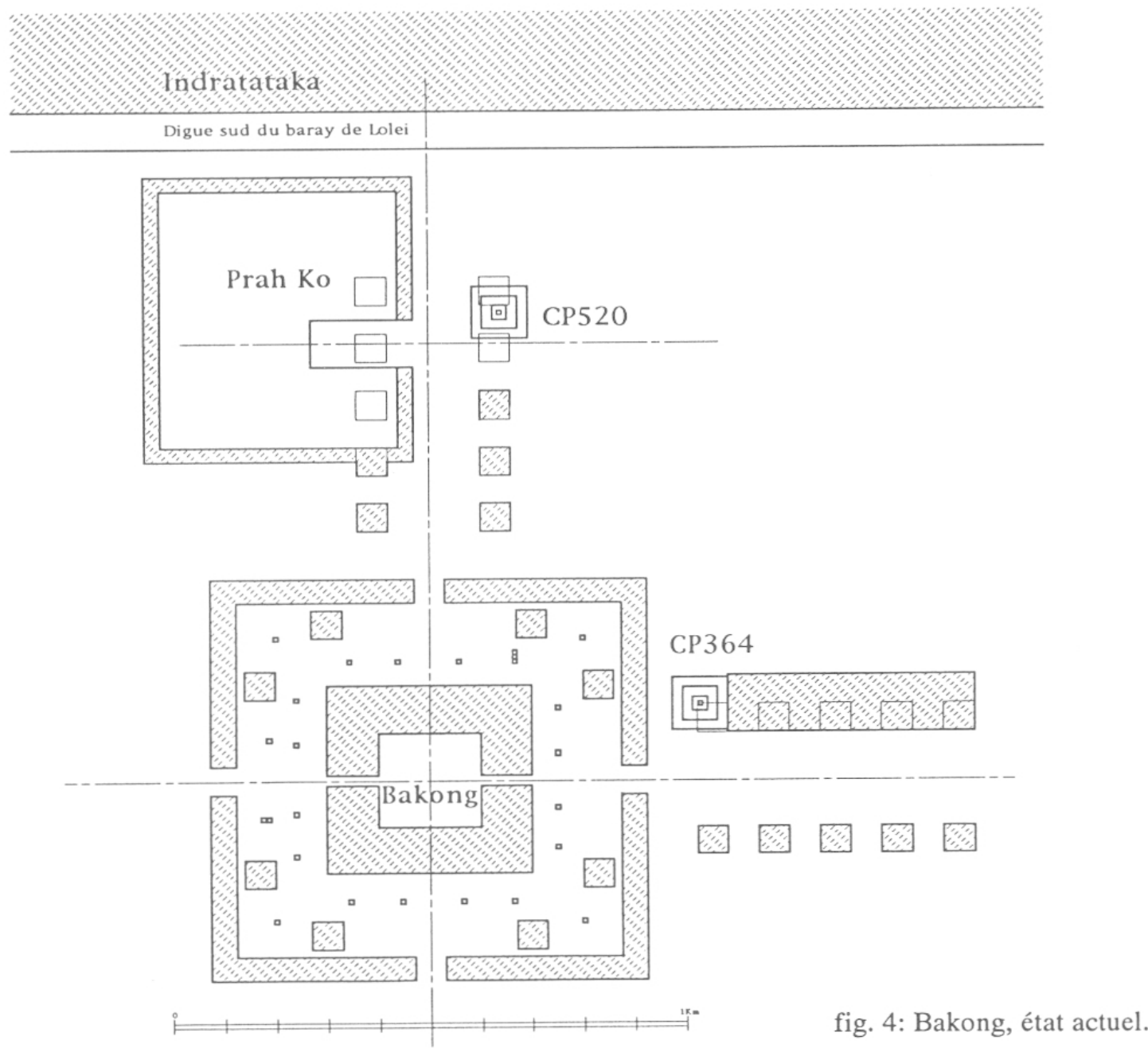

